\section{La evaluación en proyectos de extensión incluidos en el currículo universitario}

Alicia R. W. de Camilloni

Profesora Emérita de la Facultad de

Filosofía y Letras de la Universidad

de Buenos Aires, Argentina.

Miembro Honorario de la Academia

Nacional de Educación, Argentina.

acamilloni@arnet.com.ar
Integración de la docencia y la extensión /

Perspectivas

RECEPCIÓN: 24/06/16

ACEPTACIÓN FINAL: 03/10/16

\section{Resumen}

La evaluación de proyectos de extensión incluidos en el currículo universitario con propósitos académicos, profesionales y sociales articulados y un enfoque pedagógico de educación experiencial, es un proceso particularmente complejo dadas las múltiples funciones desplegadas por la evaluación en los planos institucional y estratégico. Deben tomarse decisiones en cada una de las etapas de planificación, implementación y finalización del proyecto con la intervención necesaria de los diversos actores que en él participan. En tanto el proceso de evaluación esté entramado con las acciones que se realizan en cada etapa y se resuelvan adecuadamente los problemas técnicos, sin dejar de lado los valores que se ponen en juego en el proyecto de extensión, la evaluación es un recurso que contribuye a enriquecer y profundizar los alcances y logros del proyecto y contribuye a la formación de todos los que en él participan.

Palabras clave

- Proyectos de extensión

- Educación experiencial

- Evaluación de proyectos

- Evaluación de aprendizajes

- Instrumentos

- Indicadores

\section{Resumo}

A avaliação de projetos de extensão incluídos no currículo universitário com propósitos acadêmicos, profissionais e sociais articulados e um enfoque pedagógico de educação experiencial, é um processo particularmente complexo devido às múltiplas funções desdobradas pela avaliação nos âmbitos institucional e estratégico. Devem tomar-se decisões em cada uma das etapas de planejamento, execução e finalização do projeto com a intervenção necessária dos diversos atores que nele participam. Enquanto o processo de avaliação esteja entrelaçado com as ações que se realizam em cada etapa e se resolvam adequadamente os problemas técnicos, sem deixar de lado os valores que entram em jogo no projeto de extensão, a avaliação é um recurso que contribui para enriquecer e aprofundar as transcendências e os feitos do projeto e contribui com a formação de todos os que nele participam.

Palavras-chave

- Projetos de extensão

- Educação experiencial

- Avaliação de projetos

- Avaliação de aprendizagens

- Instrumentos

- Indicadores
Para citación de este artículo

Camilloni, A. W. de (2016). La evaluación en proyectos de extensión incluidos en el currículo universitario.

En Revista +E versión digital, (6), pp. 24-35. Santa Fe, Argentina: Ediciones UNL. 


\title{
66
}

\author{
La evaluación de los aprendizajes \\ de los alumnos en un proyecto de \\ extensión plantea retos nuevos a los \\ docentes y a los propios estudiantes
}

\section{Introducción}

En un proyecto de extensión universitaria que se incluye en el currículo, como expresión de la voluntad de articular las misiones de docencia, investigación y extensión, propias de la universidad, la evaluación constituye una función que excede su aparente función de auxiliar de quienes ejercen la dirección del proyecto o de las autoridades, que la suelen emplear como fundamento de las decisiones de mayor o menor alcance que la aprobación y la marcha del proyecto exigen. Sus funciones son múltiples y no se limitan a producir un juicio de valor final sobre los alcances y virtudes del proyecto sino que sirven de recurso para enriquecerlo, dar voz a todos los participantes, replantear sus objetivos y ampliar el significado de las acciones que se emprenden.

Procuraremos presentar aquí a la evaluación como un amplio conjunto de los dispositivos que integran la estructura propositiva y accional implicada en todo proyecto y en el sistema que lo incorpora en virtud de un complejo de propósitos pedagógicos, académicos, profesionales y sociales.

En algunos aspectos, la evaluación de los aprendizajes cuenta con una amplia base teórica, en gran medida basada sobre investigación que se realiza desde larga data, así como con multiplicidad de técnicas y experiencias. Muy asociada a los aprendizajes académicos, esta evaluación, en nuestro caso, sólo permite evaluar parcialmente los aprendizajes de los alumnos en su trabajo en un proyecto de extensión. Hay rasgos relacionados con la acción, la iniciativa, la curiosidad, las muy diversas formas de razonamiento y resolución de problemas, con los atributos personales, con la interacción con otras personas, además, en escenarios nuevos, entre otros, que sólo se suelen evaluar de manera muy indirecta cuando se trata de aprendizajes académicos. La evaluación de los aprendizajes de los alumnos en un proyecto de extensión plantea retos nuevos a los docentes y a los propios estudiantes que participan en estas actividades. Pero los aprendizajes de los estudiantes, si bien son el eje de la inclusión curricular de los proyectos, están lejos de agotar los objetos de evaluación de los componentes del proyecto. Como veremos, nos proponemos añadir la evaluación de la actividad de los docentes, la evaluación de la calidad de los productos y/o servicios prestados, la de la relación de la universidad con la comunidad y, por último, la de la institucionalización de la extensión en la universidad. Cada una de estas dimensiones presenta sus peculiaridades y ha sido objeto de estudio e investigación más recientes, con la habitual declaración que acompaña a estos trabajos en el sentido de que es necesario desarrollar nuevas investigaciones y trabajar con nuevos casos para llegar a algunas conclusiones más certeras o válidas. No es este, por tanto, un campo simple de abordar ya que, a la complejidad que deviene de su carácter multifuncional debemos sumar que se trata de un campo en desarrollo y en el que no hallamos controversias sino más bien sugerencias u observaciones diversas.

En algunos aspectos, la evaluación de proyectos presenta opciones tanto para quienes están preparando la presentación de un proyecto cuanto para las autoridades que intervienen en procesos de aprobación de la propuesta. Las evaluaciones ex-ante y ex-post poseen características y propósitos diversos. En la exante se evalúa un proyecto con anterioridad a su realización. Para los diseñadores es fundamental revisar cuidadosa y rigurosamente la propuesta antes de presentarla e introducir los cambios que resulten necesarios de acuerdo con la evaluación efectuada. Para las autoridades, suele ser una modalidad obligada para dar curso a la aprobación del trabajo a realizar. En este caso, la evaluación externa puede tener carácter definitivo o constituir una instancia que da lugar a correcciones, adecuaciones o reestructuraciones del proyecto. La evaluación ex -post, que estudia el proceso y el producto del proyecto o sólo el producto, puede ser realizada también tanto por los responsables de la puesta en práctica del proyecto como por las autoridades que deben dar su aprobación y decidir, si correspondiere, sobre la iniciación, la continuidad, la interrupción o la finalización del proyecto. Los dos niveles de evaluación, el primero, institucional y el segundo, estratégico, en virtud de que persiguen diferentes objetivos, requieren la definición de indicadores específicos para ese nivel (Hart, 2011:34-58). 


\section{4}

las decisiones que resultan fundadas en los

resultados de la evaluación muestran que

encierra el ejercicio de mecanismos de poder,

en el interior y en el exterior del proyecto

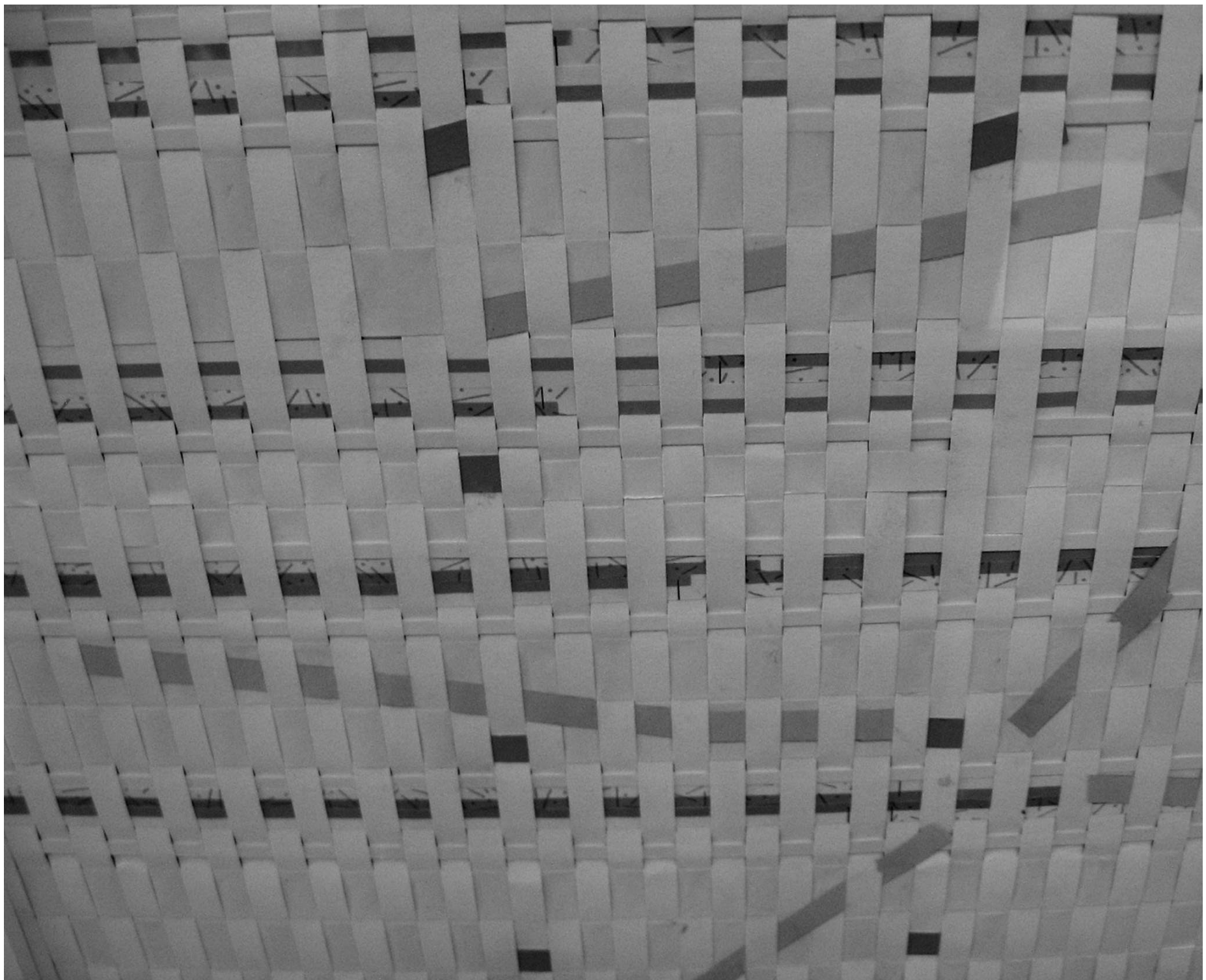

() Jorge Malachesky 
Las decisiones que resultan fundadas en los resultados de la evaluación, previa o posterior respecto de las tareas realizadas, muestran que la evaluación encierra el ejercicio de mecanismos de poder en ambos, en el interior y en el exterior del proyecto. Esta capacidad de fundar decisiones exige del evaluador cuidadosa consideración de todos los aspectos significativos del objeto a evaluar y transparencia, respeto, equidad y justificación de interpretaciones y resoluciones. Con frecuencia se recurre a mediciones en apariencia científicas, basadas en una presunta" "cientificidad" de los resultados que se deriva de la mera cuantificación de impactos y efectos. No siempre la racionalización de las mediciones asegura que la evaluación sea completa, dado que en estos proyectos, los aprendizajes y las vivencias de los participantes son un componente de gran importancia y, probablemente, no sean siempre cuantificables. Una cuestión de importancia es, pues, decidir quién será el responsable de la evaluación. Si nos preguntamos quién evalúa. Las respuestas posibles son tres: evaluación interna que realizada por las personas que llevan adelante el proyecto o por la propia institución, personas que pueden estar trabajando en el mismo aspecto evaluado o no; evaluación externa (evaluación por contrato) realizada por personas que no integran el personal del proyecto y que no pertenecen a la institución o, finalmente, una complementación de evaluación interna y externa.

En cuanto a su relación con el cronograma del proyecto, la evaluación se puede hacer de manera continua o en forma periódica.

Para que reúna las condiciones que le permitan cumplir cabalmente con sus funciones, el programa de evaluación debe ser útil, factible, apropiado, exacto y sensible, cuidadoso y respetuoso.

Las preguntas que presiden su diseño son: ¿Para quién es la evaluación? ¿Qué se ha aprendido de la evaluación? ¿Qué uso se hará de ella?

\section{La pedagogía de la educación experiencial}

En este enfoque pedagógico no se enseña de la misma manera que en la enseñanza que se limita al aprendizaje de información, que se preocupa sólo por la dimensión intelectual del aprendizaje, que no se preocupa por la práctica en el uso del conocimiento y que no relaciona el conocimiento con la acción en situación, especialmente, cuando se trata de la actuación del estudiantes en situación real, auténtica.

Así, pues, la educación experiencial, en la que se inscribe la función pedagógica de la inclusión curricular de la participación de los alumnos en proyectos de extensión, es más que el aprendizaje de información, es más que aprendizaje intelectual, se preocupa por la práctica en el uso del conocimiento y relaciona el conocimiento con la acción en situación.

Cuando se diseña un proyecto de inclusión de la educación experiencial en el currículo universitario son requisitos fundamentales a tener en cuenta, que no debe agregarse como un componente fragmentario más en el programa de formación y que es necesario estudiar cuidadosamente cuáles son las vinculaciones de este componente con los otros componentes del currículo. Este último exige la adopción de una mirada integral sobre el programa que habrán de desarrollar los estudiantes en su trayecto de formación. Cada uno de los componentes del currículo es esencial en este proceso y cada uno de ellos, merced a su heterogeneidad, aporta elementos diferentes que se vuelven insustituibles para la composición de un amplio campo de experiencias personales y profesionales de aprendizaje. Incluir la educación experiencial en el currículo universitario de un modo que resguarde su propósito pedagógico, académico y profesional, requiere proceder de acuerdo con los siguientes principios:

- No otorgar créditos académicos por el servicio sino por los aprendizajes que se espera que el alumno logre, por cuanto su inclusión curricular responde a la concepción de que el estudiante aprende de su experiencia. No es un requisito burocrático más, que se introduce en el plan de estudios sino que se concibe como un conjunto de episodios en la historia no solamente escolar sino personal del alumno. En ellos se forma como persona, como ciudadano actual y como futuro profesional.

- Por estas razones, el proyecto en su programación, implementación y evaluación debe preservar en todas sus etapas e incidentes la exigencia de rigor académico - profesional y ético - Con este propósito y como sustento de las decisiones que se proceda a adoptar, constituye un recurso importante enunciar en el proyecto con claridad los objetivos de aprendizaje de los alumnos, evitando que se pierdan en el conjunto de tareas que en estos proyectos es habitual que se entramen y se sucedan con la participación de diferentes actores en distintas circunstancias. - Cada proyecto plantea situaciones específicas y tareas con finalidades diversas. Si se las concibe como oportunidades de aprendizaje para los estudiantes, en el proyecto se habrán de prever las modalidades de enseñanza apropiadas de los conocimientos y habilidades necesarias para la realización de las tareas comprometidas en el proyecto.

- Los aprendizajes de los alumnos deben constituirse al modo de caminos inacabados que conducen a su posible inserción en variadas e imprevisibles situaciones. Con ese fin y para facilitar la movilización futura de los aprendizajes actuales, se habrá de prever condiciones para que el estudiante recupere de modo consciente, mediante la reflexión sobre lo actuado, los aprendizajes que realiza a través de esta particular experiencia

- Su mayor valor pedagógico reside en la vinculación exitosa y natural de los dos aprendizajes: el que realiza el alumno en sus actividades habituales como estudiante en la facultad, con el que realiza en el proyecto de educación experiencial, sea en las modalidades de voluntariado, aprendizaje-servicio o práctica profesional. El enlace entre ambos debe ser estimulado por los docentes. 
- Tratándose de actividades en situaciones auténticas, de la vida real, en el proyecto se debe prever la existencia de situaciones de incertidumbre en las que el equipo y el alumno tendrán que tomar nuevas decisiones, añadidas o en reemplazo de las programadas previamente.

\section{La evaluación en la educación experiencial}

En el entendimiento de que la educación experiencial asume un fundamento y una práctica diferentes de las que habitualmente se encuentran en las aulas universitarias, debemos partir de la idea de que si no se enseña de la misma manera que en ellas, no se deben evaluar los aprendizajes de la misma manera. El trabajo de extensión plantea una salida de la cotidianeidad del aula o el laboratorio. De ahí que nos preguntemos qué teoría se adopta como fundamento de las variadas decisiones que se deben tomar en lo que se refiere a la evaluación de las diversas dimensiones, componentes y resultados del proyecto de extensión. Es objeto de nuestras interrogantes iniciales la necesidad de encontrar respuestas a las siguientes cuestiones:

¿Cuál es el propósito con el que se encara aquí la evaluación? ¿A quiénes están destinados los informes de evaluación? ¿Existe un requerimiento formal de evaluación del proyecto? ¿Cuáles son los aspectos del proyecto que son más importantes de evaluar? ¿Qué objetivos tiene el proyecto? ¿Qué consecuencias deseables y no deseables podría tener?

Responder a estas preguntas nos lleva a considerar algunos de los temas que definen los mejores usos que se pueden dar a la evaluación en proyectos de extensión. En primer lugar, la determinación de quiénes son los destinatarios del informe de evaluación; en segundo lugar, como lo afirmamos antes, cuáles son los aprendizajes de los alumnos que proponemos lograr en primera instancia; en tercer lugar, qué actividades se programan para los docentes que participan en el proyecto; en cuarto lugar, cuáles serán los indicadores de la calidad de los productos y/o servicios que se producirán mediante las actividades desarrolladas en el proyecto, en quinto lugar, cómo definimos la relación que desaseamos establecer entre el grupo de trabajo y la universidad con la comunidad y, por ende, que grado y tipo de institucionalización de la extensión queremos dar al trabajo que emprendemos en este proyecto.

Como siempre que se incluyen dispositivos o instancias de evaluación en la programación e implementación de actividades educativas, es menester tener presente que la evaluación es un complejo proceso destinado a recoger información, construir juicios de valor y orientar la toma de decisiones destinadas a corregir acciones y resultados que conviene que sean reemplazados por otros preferentes, perfeccionar lo que se ha realizado o lo que está en curso de realización y prevenir la ocurrencia de acciones o efectos indeseados. En atención a la variedad de funciones que la evaluación contribuye a plasmar en los programas educativos, se han diferenciado clases o tipos de evaluación de acuerdo con la función que principalmente desarrollan en cada caso.

\section{Funciones de la evaluación de proyectos}

Como señalamos, las funciones que desempeña la evaluación son variadas.

\subsection{Evaluación para la planificación del proyecto}

La evaluación diagnóstica está destinada a efectuar un estudio de las condiciones que caracterizan la situación y los problemas con los que se ha de trabajar. Se define el problema, se trata de determinar sus causas y los factores sobre los que se puede actuar. Se procura lograr un diagnóstico realista, operativo, que sea útil para programar las acciones y determinar los recursos necesarios para ejercer la intervención. Se describen circunstancias y dificultades, características de la población con la que se trabajará, requisitos de conocimientos y habilidades que serán necesarios en docentes y alumnos para realizar la intervención.

Entre los instrumentos que se utilizan para la evaluación diagnóstica y la planificación del proyecto mencionamos los siguientes:

- Registros anteriores sobre el problema con el que se va a trabajar

- Diagnósticos y opiniones de los participantes

- Encuestas y entrevistas individuales y en grupos focales a beneficiarios/usuarios y a miembros de la comunidad.

La evaluación preventiva es una evaluación inicial de riesgos que facilita la formulación del proyecto identificando en la etapa de programación aquellos indicadores que podrían señalar condiciones frente a las cuales convendrá adoptar medidas especiales para evitar efectos no deseados y redireccionar, si es necesario, las acciones del proyecto.

La evaluación prospectiva estima el valor del logro de los objetivos del proyecto antes de que éste se lleve a cabo. Se utilizan indicadores de impacto destinados a demostrar si la propuesta puede aportar a la solución del problema identificado y definido. Se fundamenta la decisión sobre el tipo de intervención elegido. Como veremos, se emplean técnicas de análisis de costos o análisis de costo-beneficio, entre otras.

El Estudio de viabilidad permite estimar si el proyecto elaborado es posible de implementar y completar y si ha de llevar a lograr sus propósitos. Las acciones que componen el estudio son "recopilar información suficiente para:

- Identificar las limitaciones, restricciones y supuestos.

- Detectar las oportunidades.

- Analizar el modo actual de funcionamiento de la organización. 
- Definir los requisitos que configuran el proyecto.

- Evaluar las distintas alternativas.

- Llegar a un acuerdo sobre la línea de acción."

En tanto, el Estudio de factibilidad de un proyecto se centra en los costos y efectos económicos del proyecto.

\subsection{Evaluación para el control}

La evaluación cumple una función reguladora cuando hace un seguimiento continuo de la marcha del proyecto. El plan de evaluación establecido en la etapa de programación sirve de marco y guía para el monitoreo y la observación de acciones y efectos durante la implementación. El plan de evaluación debe ser adecuado y reajustado cuando la realidad del funcionamiento del proyecto lleva a introducir modificaciones en el programa original del proyecto. La regulación, en consecuencia, tiene una capacidad limitada sobre el proyecto. Lo regula en tanto se mantiene sin cambios el programa original.

La evaluación realizada sobre la base de un programa de control de calidad debe tener en cuenta que la calidad es multidimensional y dinámica. Tiene que trabajar con un número importante de variables diferentes, entre ellas, las que definen calidad social y calidad técnica de los logros del proyecto. Un recurso interesante para implantar un programa de control de calidad consiste en la construcción participativa con los diferentes actores que intervienen en el proyecto, de un modelo que estructure los aportes que el proyecto recibe de esos diversos actores, del exterior del escenario del proyecto, de la comunidad y de la universidad. Los impactos buscados se definen y se contempla en el modelo acciones e interacciones que conducen a obtener los resultados deseados. Tratándose de un proyecto que transcurre en un lapso determinado, las etapas y su relación con los niveles de calidad también deberán ser incluidas en el modelo.

Son útiles los diagnósticos y opiniones anteriores de los participantes y las encuestas y entrevistas individuales y en grupos focales a beneficiarios/usuarios y a miembros de la comunidad (antes y después).

1) Estudio de viabilidad de un proyecto: cómo y por qué llevarlo a cabo" Project Management. OBS. Universita de Barcelona. http://www.obs-edu. com/es/blog-project-management/ causas-de-fracaso-de-un-proyecto/ estudio-de-viabilidad-de-un-proyectocomo-y-por-que-llevarlo-cabo

\subsection{Evaluación para la mejora}

La información recogida mediante los diferentes instrumentos de evaluación permite adoptar decisiones adecuadas durante la implementación, no sólo en términos de control del cumplimiento de acciones y objetivos previstos sino con fines de mejoramiento de las actividades y de reelaboración de objetivos. La evaluación contribuye, de esta manera, a rediseñar el proyecto con el fin de obtener mejores resultados en lo que se refiere a aprendizajes y a bienes o servicios producidos. La evaluación sirve a propósitos de mejoramiento porque tiene capacidad para:

- Proveer información.

- Clarificar metas.

- Servir de estímulo.

- lluminar los procesos.

- Ofrecer términos de comparación con otros programas o instituciones.

- Favorecer la innovación.

\subsection{Criterios de evaluación del proyecto}

La elección y el acuerdo sobre los criterios que se usarán en la evaluación constituye una de las cuestiones que adquieren la mayor importancia en los procesos de evaluación y, en la medida en que, como antes dijimos, están entretejidas las operaciones de evaluación con las actividades del proyecto, tienen, de hecho, una función regulatoria sobre él. Aunque los criterios de evaluación se establecen de manera ad-hoc para cada proyecto, tratándose de actividades que tienen un fin social, es posible mencionar algunos que son frecuentemente enunciados por los diseñadores de los proyectos. Entre ellos:

- Cobertura: número de beneficiarios.

- Consistencia entre objetivos y metas programados y logros obtenidos (eficacia).

- Calidad del servicio (mayor... menor) (cómo).

- Buen uso de los recursos (eficiencia).

- Replicabilidad del proyecto.

- Aprendizajes realizados.

- Resultados de corto plazo-largo plazo.

\section{La evaluación del logro de los propósitos del proyecto} Para evaluar el logro de los propósitos del proyecto es preciso adoptar una perspectiva muy amplia y abarcadora de los múltiples objetivos y funciones, actividades y tareas, personas con diferentes roles y niveles de responsabilidad e intereses se han puesto en juego en la marcha del proyecto.

En relación con los logros del proyecto, cinco dimensiones principales deben ser evaluadas:

1) Aprendizajes de los estudiantes: actitudes, competencias $y$ conocimientos, compromiso social y ciudadano, plan de carrera. 
2) Desarrollo profesional y académico de los docentes.

3) Satisfacción y aprendizajes de la comunidad atendida.

4) Desarrollo de los organizadores.

5) Calidad del servicio.

Están implicados en el proceso de evaluación todos los que participan en el proyecto, docentes, estudiantes, beneficiarios/usuarios directos e indirectos, administradores, autoridades de la unidad académica $y / o$ de la universidad, miembros de la comunidad.

\section{La planificación de la evaluación}

El cumplimiento de los requisitos que hemos ido enumerando requiere que las acciones de evaluación estén planificadas, concertadas y establecidas en cronograma. Los instrumentos deben ser construidos y administrados. Los tiempos necesarios para cumplir con las tareas de la evaluación deben ser previstos y las tareas cuidadosamente distribuidas, En muchos casos deberán ser explicadas a quienes provean la información necesaria para la evaluación.

Destacamos, pues, las tareas que se incluyen en esta planificación.

- Organizar el trabajo.

- Sistematizar los pasos.

- Asignar tiempo a las tareas.

- Asignar responsabilidades.

- Proveer los recursos.

- Determinar qué tipo de informe de evaluación se espera producir.

\subsection{Fases del proyecto y evaluación}

- Fase previa: Estudio de viabilidad (y, si es necesario, de factibilidad).

- En el curso de la implementación: evaluación o monitoreo formativo de las operaciones del programa para efectuar los ajustes necesarios. - Evaluación final: control sobre los logros y su relación con los fines y objetivos iniciales y los que surgieron en el desarrollo del proyecto. - Seguimiento: evaluación en el período posterior (corto, mediano y largo plazo) a la implementación del proyecto para controlar cuáles son sus efectos, su duración y sus posibles consecuencias.

Es imprescindible adecuar las expectativas de la planificación de la evaluación a los recursos disponibles. Si no los hubiera, deberán integrar la asignación de parte de recursos previstos para la implementación del proyecto.

Analizar la disponibilidad de recursos de personal: capacitado, no capacitado en evaluación, de apoyo, administrativo y profesional. Igualmente, con respecto a recursos de tiempo: estimar el tiempo necesario y disponible, los momentos de urgencia según el desarrollo del proyecto, el tiempo del personal asignado.
Inventariar los recursos de equipamiento necesarios para la evaluación de la calidad del producto y/o la calidad del servicio. Estimar los recursos de dinero necesarios para efectuar la evaluación. Determinar cuáles han de ser los recursos informáticos necesarios para registrar, procesar e interpretar datos.

En cuanto a los métodos o modelos de evaluación empleados, la experiencia en este terreno muestra que se usan métodos cuantitativos, métodos cualitativos y métodos cuanti-cualitativos.

\section{Instrumentos para recoger información sobre el trabajo del equipo docentes-estudiantes de la universidad}

Los instrumentos que se emplean para recoger información dependen del proyecto específico de que se trate. Son muchos los que están disponibles. Cada uno de ellos responde a ciertas pautas para su construcción, administración e interpretación de la información obtenida. Entre los que son habitualmente utilizados encontramos:

- Documentos del proyecto.

- Estadísticas.

- Actas de reunión.

- Observación.

- Informes.

- Encuestas a estudiantes, docentes, autoridades.

- Entrevistas individuales a estudiantes, docentes, autoridades.

- Grupos focales con estudiantes y docentes.

- Diarios de participantes (docentes y estudiantes).

- Evaluaciones de aprendizajes de participantes docentes y estudiantes:

- Evaluación de actitudes.

- Evaluación de desempeño.

- Evaluación de conocimientos.

\section{Sobre los roles de los estudiantes y sus evaluaciones}

La atribución de roles a los estudiantes es la decisión con efectos pedagógicos, académicos y profesionales más importante entre las que están implicadas en el diseño de un proyecto de aprendizajeservicio. Hay algunas precauciones que se han de tomar para que estas decisiones sean adecuadas a sus fines: definir los diferentes roles muy claramente y discutirlos con estudiantes y socios, preparar a los estudiantes para el aprendizaje-servicio: conocer el lugar, las personas, el ambiente y situación que encontrarán y características y alcance de la necesidad a ser satisfecha y explicarles el sentido social y personal del proyecto.

Se tendrá en cuenta que el aprendizaje de los estudiantes depende de dos condiciones básicas. La primera es que tiene que saber 
qué va a aprender y no solo qué debe hacer. Y la segunda es que debe aceptar que en la realización de las tareas planteadas por el proyecto puede lograr aprendizajes ligados a la formación académica y profesional que busca en la universidad.

La evaluación, en lo que respecta a los estudiantes, cubre, en los términos en que ha sido diseñado el proyecto, tres dimensiones: el trabajo académico, el desarrollo personal y el servicio.

\section{La Escala de experiencialidad}

Maurice Gibbons y David Hopkins (1980) construyeron una Escala de experiencialidad que ha tenido mucha difusión porque ofrece una base para distinguir niveles de aprendizaje experiencial en la evaluación. Diferenciaron cinco modos de experiencia y nueve niveles, de menor a mayor compromiso con la experiencia según quiere o procura.

\begin{tabular}{|c|c|c|}
\hline Modo & Nivel & \\
\hline \multirow[t]{2}{*}{ Receptivo } & 1 & Estimulado: observa filmes, TV, video, etcétera \\
\hline & 2 & Espectador: observa el hecho real en su contexto normal \\
\hline \multirow[t]{2}{*}{ Analítico } & 3 & Exploratorio: juega, ensaya, explora y pone a prueba \\
\hline & $\overline{4}$ & Analítico: estudia y experimenta sistemáticamente \\
\hline \multirow[t]{2}{*}{ Productivo } & 5 & Generativo: crea, organiza, construye, teoriza \\
\hline & 6 & Desafío: tareas difíciles pero deseables de cumplir \\
\hline \multirow[t]{2}{*}{ De desarrollo } & 7 & Competencia: ser hábil en actividades importantes \\
\hline & 8 & Dominio: alto estándar de calidad en el desempeño \\
\hline Personal & 9 & Conocimiento personal: Busca excelencia y madurez \\
\hline
\end{tabular}

Con el reconocimiento de que la variedad de clases de aprendizajes es un rasgo típico de los proyectos de aprendizaje-servicio, el plan de evaluación de lo experimentado y ganado por los estudiantes, debe incluir un abanico amplio de instrumentos de evaluación. Se tendrá en cuenta, por lo tanto, que es preciso evaluar el conocimiento adquirido con más de una modalidad de evaluación. Dados los objetivos propios del aprendizaje-servicio, se debe evaluar el trabajo en grupo, coevaluar con el tutor del servicio la calidad de éste, sus procesos y resultados, el desarrollo personal del alumno mediante autoevaluación, evaluación por pares y evaluación de actitudes. Especial atención corresponde poner a: 1) El trabajo en el campo: se puede resolver mediante evaluación por los dos tutores con coloquio, informe, portafolio.

2) El diseño e implementación de proyectos por medio de la evaluación del trabajo final o portafolio.

3) La presentación de un caso trabajado con desarrollo escrito u oral.
4) La resolución de problemas reales en situación mediante la observación, la autorreflexión y la autoevaluación en tarea grupal o individual.

En suma, es prioritario que la evaluación contribuya a:

- Comprobar si los estudiantes poseen los conocimientos y habilidades necesarias para desarrollar las actividades de servicio. - Enseñarles a reflexionar continuamente sobre la práctica y sus resultados.

- Brindarles orientación para la planificación de su trabajo y estudio.

Como hemos visto, la participación en proyectos de extensión en los que la educación experiencial coloca a los alumnos en situaciones de trabajo real es especialmente adecuada para realizar lo que se denomina "evaluación de desempeño". Ésta requiere, precisamente, que en lo posible los evaluadores adopten una modalidad que posee ese objetivo específico: recoger evidencias sobre respuestas auténticas en situaciones de la vida real. Estas actuaciones permiten demostrar los saberes en la práctica y la evaluación adquiere así mayor validez y se compara el desempeño del alumno con modelos de estándares en la realización de la tarea. Para acrecentar la confiabilidad de las evaluaciones cualitativas se usan rúbricas de evaluación. Con ese fin, se definen las áreas de habilidad que se van a evaluar, se selecciona el tipo de instrumento de evaluación, se construyen la tarea de evaluación y la rúbrica y se definen los estándares de desempeño. Es menester validar las mediciones, administrar, calificar, interpretar e informar los resultados. Esta modalidad de evaluación requiere, ciertamente, tiempo y recursos; y, aunque no es fácil llevarla a cabo, es necesaria en los programas de formación profesional.

Un aspecto particularmente delicado pero específico del trabajo en aprendizaje-servicio es la evaluación de las actitudes prosociales de los estudiantes y de los docentes participantes.

\section{Las actitudes prosociales y las destrezas}

Enumeramos algunas de estas actitudes que podrían evidenciarse en situaciones reales de trabajo en el proyecto: dar ayuda física a otro mediante una conducta no verbal, contribuir a la mejora de condiciones ambientales para la acción propia y de otros, dar y compartir, brindar ayuda verbal a otros por medio de explicación o instrucciones, ofrecer consuelo verbal, demostrar valoración del otro, manifestar una escucha profunda, empatía, solidaridad, servicio en interés del otro, integridad, conciencia cívica.

En el trabajo también podrá evidenciarse la posesión de variadas destrezas: de pensamiento, de observación, de interacción social de planificación, para reunir información, para resolver problemas, 
para tomar decisiones, para autoevaluarse, para revisar lo hecho y corregirlo, para negociar y para mediar en situaciones de conflicto.

\subsection{La evaluación de la educación experiencial durante la actividad}

Es importante recoger información sobre las actividades y las reflexiones de los estudiantes en tanto están desempeñándose como miembros responsables en el proyecto. Diversos recursos se usan con este propósito. Indicamos los que se emplean con mayor frecuencia:

- Cuadernos de bitácora de los alumnos.

- Diarios de los alumnos.

- Reuniones semanales de reflexión y discusión.

- Observaciones de las acciones de los alumnos.

- Informes de autoevaluación.

- Exámenes de conocimientos individuales.

- Trabajos grupales: productos, proyectos, acciones.

\subsection{La evaluación de los aprendizajes después de concluir la actividad}

Al concluir la actividad, el equipo docente, el grupo de pares y el estudiante harán una evaluación de los aprendizajes realizados en términos de conocimientos, destrezas y actitudes prosociales. Si fuera necesario, según la índole del trabajo efectuado y su vinculación con una asignatura determinada, podría corresponder la administración de un instrumento de carácter académico para la evaluación de los aprendizajes de la asignatura o de las competencias profesionales adquiridas.

\subsection{La evaluación por los tutores}

Es función de los tutores, además de la tarea de acompañamiento, ayuda y protección, evaluar las capacidades adquiridas por los estudiantes, para lo cual deberán desarrollar en ellos mismos la capacidad para planificar y ejecutar la evaluación de los alumnos en la práctica, acordar con los éstos el plan y las técnicas de evaluación y revisar el plan durante la actividad, cuidar que las evaluaciones estén basadas en evidencias y puedan ser claramente explicadas y cuidar de que las evidencias sean válidas y confiables. En las tareas de evaluación, los tutores deberán observar las acciones y resultados, usar diversas fuentes de información, estimular a los estudiantes para que se autoevalúen , brindar retroalimentación y proalimentación permanentemente y hacer seguimiento de los efectos de sus orientaciones, buscar retroalimentación en las opiniones y experiencias de los beneficiarios/usuarios, mostrar a los estudiantes cómo mejorar su desempeño actual y futuro, elaborar registros e informes de evaluación, dar lugar a pedidos de revisión de las evaluaciones por los estudiantes, procurar recibir retroalimentación de los alumnos sobre su experiencia en el proyecto, buscar retroalimentación sobre el trabajo del tutor, recoger retroalimentación del alumno sobre la evaluación implementada y, por cierto, autoevaluarse.

Para apoyar el trabajo de los tutores es siempre conveniente que los responsables del proyecto procuren orientarlos para tomar decisiones y revisar sus planes de trabajo, conversen con ellos y con todo el grupo sobre los aspectos éticos del trabajo que desarrollan los estudiantes, los alienten para organizar reuniones de discusión entre los estudiantes y, eventualmente, con otras personas (especialistas, receptores socios, etc.), les den consignas claras para el trabajo y los ayuden a administrar cuidadosamente los tiempos asignados a las tareas, les recomienden bibliografía adecuada para ellos y para los estudiantes, se mantengan en contacto con los tutores o supervisores del lugar donde se brinda el servicio, colaboren con los tutores en el monitoreo del desempeño de los estudiantes, los apoyen en el desarrollo de autoconfianza en los participantes para realizar su tarea y, si fuera necesario elaboren con los tutores planes contingentes.

\section{Evaluación de la institucionalización de la educación experiencial en la universidad}

La incorporación curricular de la educación experiencial, su reconocimiento como actividad formadora, articulada con las misiones de docencia e investigación y las relaciones que se establecen de manera sistemática con la comunidad responden a decisiones estratégicas de la institución. Su continuidad requiere el apoyo de la universidad, que debe adoptar nuevas reglamentaciones, medidas, incentivos y acciones que consoliden cotidianamente esta línea de trabajo.

Atento a que es una condición necesaria para hacerla posible, el compromiso que asume la universidad es también objeto de evaluación.

Angie Hart (2011:37) cita un trabajo de Oliver et al. (2008) en el que se explicitan los aspectos a considerar en este caso. Los señalados son los siguientes: "se evalúa el compromiso organizacional (liderazgo, estructuras, recursos y actitudes) de la universidad con la comunidad, los procedimientos empleados, las relaciones interpersonales (honestidad y competencia), el impacto (aprendizaje mutuo y acción social), la influencia sobre la agenda de investigación".

No es éste un trabajo sencillo para una evaluación comprometida con los valores que se postulan en esta relación, por cuanto la medición del nivel de compromiso de la universidad con la sociedad se manifiesta a través de interacciones que ocurren en diferentes niveles y unidades de la institución y que tienen una gran heterogeneidad y gran número de actores.

Los principios de estas interacciones requieren que todos los participantes posean capacidades para escuchar, comprender, producir y transferir conocimiento a la comunidad y aprender de 
¿Para quién es la evaluación?

¿Qué se ha aprendido de la evaluación?

¿Qué uso se hará de ella?

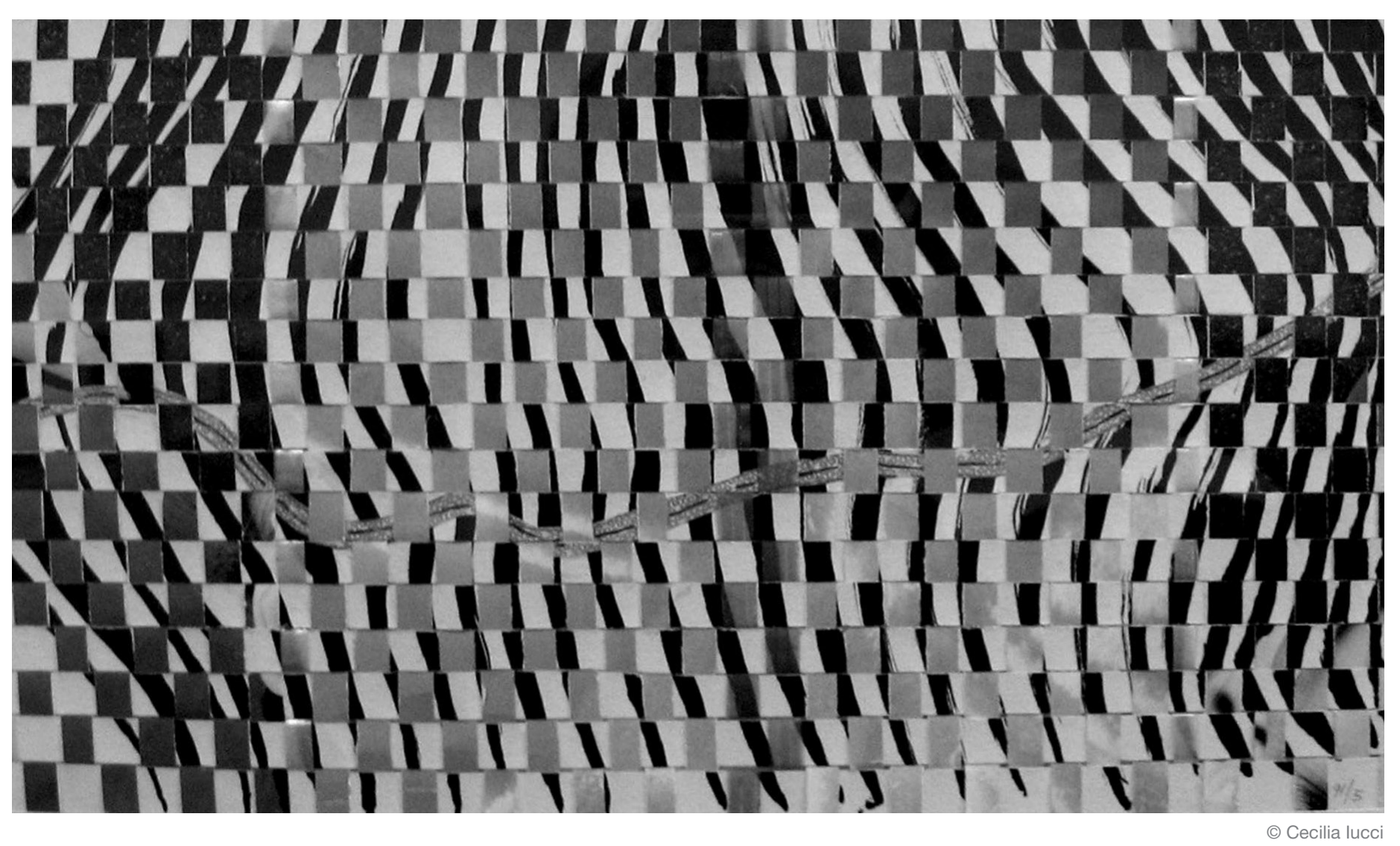


ésta, recoger información, contribuir a identificar, definir y solucionar problemas e intervenir en la definición de políticas públicas. Se identifican siete dimensiones por su significatividad en la evaluación del compromiso de la universidad con el público. Son ellas:

1) El acceso público a las facilidades de la universidad: acceso público a las bibliotecas, a los edificios, a los jardines, a las conferencias, museos, galerías de arte, deportes, a los laboratorios, a los auditorios.

2) El acceso público al conocimiento: acceso a cursos regulares, a cursos de extensión, a ferias o muestras de ciencias, tecnologías y artes, a bases de datos, a participación en investigación.

3) El compromiso de los estudiantes en las interacciones con el público: incorporación en el currículo académico de estudios sobre problemáticas sociales en la historia pasada y reciente; participación en actividades de voluntariado, aprendizaje-servicio y pasantías profesionales, promoción de actividades estudiantiles de solidaridad social.

4) El compromiso de los docentes en las interacciones con el público: participación en actividades de extensión, asesoramiento a la comunidad, trabajo en colaboración con miembros de la comunidad, investigación sobre problemas de la comunidad, conferencias públicas, enseñanza a los estudiantes para actividades de voluntariado, aprendizaje-servicio y pasantías profesionales.

5) La ampliación de la participación de todos los actores en actividades conjuntas: acciones para apoyar la solución de necesidades de la comunidad, iniciativas para promoción de acciones de innovación por miembros de la misma.

6) El apoyo para mejores logros económicos y mayor igualdad social en la comunidad: estrategias para lograr mayor matrícula de estudiantes con desventajas sociales, económicas y físicas, para mejorar la tasa de éxito de estudiantes con desventajas, brindar asistencia tecnológica, asesoría en negocios, asistencia para diseño y ejecución de proyectos, participación en la definición de políticas públicas.

7) La efectivización de relaciones institucionales y la construcción de asociaciones: creación de un órgano en la universidad/ facultad/departamento para administrar el programa, programa de investigaciones colaborativas con la comunidad, programas de redes de enseñanza y aprendizaje colaborativos con la comunidad, website con páginas de la comunidad, mesas de ayuda a la comunidad.

La institucionalización de las relaciones con la sociedad supone un proceso de construcción progresiva en el que todos aprenden, ensayan, proponen, argumentan y, en particular, escuchan. No hay medición exacta para un buen número de los indicadores; en algunos se observarán logros parciales, en otros será preciso remontar, a veces, fracasos anteriores y habrá, igualmente, programas en los que se obtengan éxitos importantes. La evaluación, en su sentido más amplio, permitirá hallar éxitos y vacíos y servirá como base de un semillero de ideas que en el espíritu de la institución universitaria encontrarán la raíz de la asociación efectiva de la universidad con la sociedad.

\section{La calidad del servicio}

Este aspecto de la tarea realizada, en conjunto con los aprendizajes realizados por los estudiantes, los docentes y los miembros de la comunidad, es la razón de ser del proyecto. El concepto de calidad es, ciertamente, polisémico, por lo que es imprescindible definir previamente y durante el transcurso de la experiencia qué características se espera que se evidencien, se trate de un producto o de un servicio. En relación con estas características, se podrá estimar el valor de la asistencia o el acompañamiento brindado. Es conveniente recordar que es el proveedor quien debe satisfacer la expectativa del cliente con un determinado nivel mínimo de calidad. Para poder evaluar la calidad de la asistencia es indispensable conocer cuál es la expectativa del cliente, si ha sido satisfecha o si el estándar previamente establecido en acuerdo entre las partes ha sido alcanzado.

Según se trate de uno u otro tipo de proyecto, las técnicas empleadas para evaluar la calidad de lo producido serán decisión de los evaluadores. En términos generales, las técnicas de evaluación de la calidad del servicio son variadas y encontramos entre ellas: las evaluaciones informales como la experiencia directa del evaluador sobre lo producido, experiencia directa, la conversación informal, los buzones de respuestas de opinión del cliente/usuario/socio, por ejemplo. Son evaluaciones formales la observación participante - cuadernos de notas de observación en el campo, la discusión en grupos focales con o sin cuestionario guía, las encuestas y las entrevistas (para las cuales se deben preparar calendario y cuestionario) y las observaciones con lista de control con base en la observación de acciones o de atributos del producto. Se usan, asimismo, estudios experimentales centrados en los resultados y estudios de casos.

Un tema de alta relevancia en la evaluación de un proyecto de extensión es el relativo a los aprendizajes y experiencias de los miembros externos, beneficiarios o usuarios a través de su participación en el proyecto. Estos aprendizajes y experiencias deben ser evaluados de manera formal o informal a como sea posible hacerlo. Asimismo, otro objeto a evaluar son los aprendizajes y experiencias del equipo del proyecto a partir de su relacionamiento con la comunidad.

En síntesis, la evaluación del programa requiere que se haga una descripción en profundidad de lo actuado con un análisis contexto espacial y temporal, a un tiempo holístico y analítico, en el que se evalúen los efectos deseados y los no deseados, en el que surja lo bueno y lo malo, De este modo, la evaluación ilumina el programa, 
trata de comprenderlo. Con ese fin, hace muchas preguntas importantes y emplea, cuando es posible, un método de estudio cuanti y cualitativo.

Entre los instrumentos utilizados se incluyen: análisis de archivos y de documentos, análisis de contenido, colección de muestras de trabajos, pruebas de laboratorio, observación independiente y participante, grupos focales, encuestas, entrevistas, relatos, foros, estudio de incidentes críticos, bases de datos, fotos, mapas y planos, estadísticas, testimonios, modelos lógicos, programas informáticos, tablas, resúmenes, noticias periodísticas, entre otros muchos.

Fundados en la información recibida y de los valores académicos, profesionales y sociales que dieron origen al proyecto, los evaluadores construyen un juicio de valor.

\section{El informe de evaluación}

La evaluación aporta conocimiento sobre la marcha del programa. El informe de evaluación reúne toda la información que resulte significativa para conocer éxitos, dificultades, problemas resueltos y no resueltos. Dará cuenta de las actividades desarrolladas y de sus resultados o productos. Un lugar principal tendrán las evaluaciones de los aprendizajes de los alumnos y las relaciones con la comunidad o el socio o socios del proyecto. La evaluación de la calidad del servicio constituye otro elemento importante del informe. Finalmente, en un informe de evaluación se hace un juicio de valor acerca de lo actuado y, si se desea continuar con las actividades, un plan de mejora si fuera necesario.
Uno de los recaudos que se deben tomar es proteger la confidencialidad de las informaciones personales y los derechos humanos de todas las personas con las que se trabaja. Es indispensable compartir la información con los participantes internos y externos. Sin embargo, a criterio de los responsables, los informes pueden elaborarse de manera diversa según sean sus destinatarios.

Así, entonces, la difusión de los resultados de la evaluación puede adecuarse conforme al informe de evaluación de los resultados para las autoridades de la facultad/universidad, para el equipo docente, para los estudiantes o para los socios del proyecto en la comunidad. No se trata de ocultar la información sino de brindar aquella que resulte más apropiada para los participantes en cumplimiento del propósito de evaluación para la mejora.

Dado que la difusión de los resultados de los trabajos es un compromiso que asumen autoridades y participantes en los proyectos, conviene preparar un artículo breve para el periódico de la facultad/universidad o videos o exposiciones fotográficas o semejantes que ayuden a comprender el significado de las actividades emprendidas.

Es también necesario recoger impresiones de docentes y estudiantes, positivas y negativas, y discutirlas con los miembros del equipo, participantes comunitarios y otros miembros de la comunidad.

$\mathrm{Y}$, finalmente, es recomendable presentar el trabajo en congresos, ateneos con colegas, y en artículos de revistas universitarias, científicas y comunitarias para compartir la experiencia en un campo en el que estas labores deben cobrar creciente impulso.

\section{Referencias bibliográficas}

Hart, A. (2011). Auditing and Evaluating University-Community Engagement: Lessons from a UK Case Study. Higher Education Quarterly 0951-5224, 65 (1, January), 34-58.

Gibbons, M. \& Hopkins, D. (1980). Scale of Experientiality. In How experiential is your experience-based program? The Journal of Experiential Education, 3 (1). 